\title{
Single-Arm Longitudinal Study to Evaluate a Decision Aid for Women Offered Neoadjuvant Systemic Therapy for Operable Breast Cancer
}

\author{
Nicholas Zdenkowski, BMed, FRACPa,b,c; Phyllis Butow, $\mathrm{PhD}^{\mathrm{d}}$; Andrew Spillane, $\mathrm{PhD}^{\mathrm{a}, \mathrm{e}}$; \\ Charles Douglas, PhDc; Kylie Snook, BMed, FRACS ${ }^{e}$; Mark Jones, BPhys ; Christopher Oldmeadow, PhD; \\ Sheryl Fewster, $\mathrm{MA}^{\mathrm{g}}$; Corinna Beckmore, $\mathrm{BSc}^{\mathrm{h}}$; and Frances M. Boyle, PhD ${ }^{\mathrm{a}, \mathrm{i}}$; \\ for the Australia and New Zealand Breast Cancer Trials Group
}

\begin{abstract}
Background: Neoadjuvant systemic therapy (NAST) is an increasingly used treatment option for women with large operable or highly proliferative breast cancer. With equivalent survival outcomes between NAST and up-front surgery, the situation-specific preference-sensitive nature of the decision makes it suitable for a decision aid (DA). This study aimed to develop and evaluate a DA for this population. Methods: A DA booklet was developed according to international standards, including information about adjuvant and neoadjuvant treatment, outcome probabilities, and a values clarification exercise. Eligible women, considered by investigators as candidates for NAST, were enrolled in a multi-institutional, single-arm, longitudinal study. Patient-reported outcome measure questionnaires were completed preand post-DA, between chemotherapy and surgery, and at 12 months. Outcomes were feasibility (percentage of eligible patients accessing the DA); acceptability to patients (percentage who would recommend it to others) and clinicians (percentage who would use the DA in routine practice); and decision-related outcomes. Results: From 77 eligible women, 59 were enrolled, of whom 47 (79.7\%; 95\% Cl, 69.4-89.9) reported having read the DA; 51 completed the first post-DA questionnaire. Of these 51,41 participants $(80.4 \% ; 95 \% \mathrm{Cl}, 69.5-91.3)$ found the DA useful for their decision about NAST. Of 18 responding investigators, 16 (88.9\%; $95 \% \mathrm{Cl}, 74.4-103.4)$ indicated they would continue to use the DA in routine practice. Post-DA, decisional conflict decreased significantly $(P<.01)$; anxiety and distress decreased significantly; and $86.3 \%(95 \% \mathrm{Cl}, 73.7-94.3)$ achieved at least as much decisional control as they desired. Conclusions: This DA was feasible and acceptable to patients and clinicians, and improvement in decision-related outcomes was demonstrated when used in combination with clinical consultations. This DA could safely be implemented into routine practice for women considering NAST for operable breast cancer.
\end{abstract}

aSydney Medical School, Faculty of Medicine, University of Sydney, Camperdown; bepartment of Medical Oncology, Calvary Mater Newcastle, Waratah; 'School of Medicine and Public Health, Faculty of Medicine, University of Newcastle, Callaghan; ${ }^{d}$ Centre for Medical Psychology \& Evidence-Based Decision-Making, School of Psychology, University of Sydney, Camperdown; 'Breast and Surgical Oncology at the Poche Centre, The Mater Hospital, North Sydney; ${ }^{f}$ Clinical Research Design and Statistics Support Unit, Hunter Medical Research Institute, New Lambton Heights; ' ${ }^{C}$ Consumer Advisory Panel, Australia and New Zealand

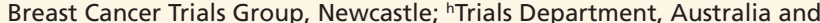
New Zealand Breast Cancer Trials Group, Newcastle; and 'Patricia Ritchie Centre for Cancer Care and Research, North Sydney, NSW, Australia. Submitted August 31, 2017; accepted for publication November 21, 2017. The authors have disclosed that they have no financial interests, arrangements, affiliations, or commercial interests with the manufacturers of any products discussed in this article or their competitors.
This work was funded by the HCF Research Foundation and by the Breast Cancer Institute of Australia as the fundraising arm of the Australia and New Zealand Breast Cancer Trials Group. Dr. Zdenkowski is supported by a Hunter New England Local Health District Clinical Research Fellowship. Drs Boyle and Spillane are supported by the Friends of the Mater Foundation. Dr. Butow has an NHMRC Research Fellowship. This study is prospectively registered on the Australian New Zealand Clinical Trials Registry (www. anzctr.org.au, ACTRN12614001267640).

Author contributions: Study concept and design: Zdenkowski, Butow Douglas, Boyle. Data acquisition: Zdenkowski, Spillane, Snook, Boyle. Data analysis and interpretation: Zdenkowski, Butow, Jones, Oldmeadow, Douglas, Boyle. Manuscript preparation: All authors. Critical revision: All authors.

Correspondence: Nicholas Zdenkowski, BMed, FRACP, Department of Medical Oncology, Calvary Mater Newcastle, Edith Street, Waratah, NSW, 2298, Australia. Email: nick.zdenkowski@newcastle.edu.au 
Patient-centered care is a key component of highquality healthcare. ${ }^{1,2}$ The process of shared decisionmaking supports patient-centered care by actively involving the patient and their healthcare providers in choosing the best treatment option. ${ }^{3}$ One strategy to implement shared decision-making is the patient decision aid (DA), which is an evidence-based, structured tool to support deliberation between patients, clinicians, and others when there is more than one treatment option. ${ }^{4,5}$

For women with large or highly proliferative operable breast cancer, the decision about whether to receive neoadjuvant systemic therapy (NAST) is guided by preferences that may lead to individual patients with similar clinical circumstances choosing different options. DAs have been shown to help guide patients in such preference-sensitive situations and improve decision-related outcomes, including reducing decisional conflict, increasing patient knowledge, increasing their involvement in decision-making, and improving their understanding of risks associated with the options. ${ }^{6}$ Several DAs have been developed to address values-sensitive patient decisions for early-stage breast cancer ${ }^{7}$; however, none was available for women considering NAST for operable breast cancer. This treatment option involves a complex interplay of preferences, ${ }^{8}$ presented in the context of a distress-inducing diagnosis of cancer. Despite this distress and decisional complexity, previous work has shown that patients benefit from being involved in decisions about management of early-stage breast cancer. ${ }^{9}$

An increasing proportion of patients with operable breast cancer are being treated with NAST. ${ }^{10}$ Advantages include downstaging from mastectomy to lumpectomy and downstaging the axilla ${ }^{11,12}$; better oncologic clearance, allowing for consideration of immediate breast reconstruction in women still requiring mastectomy; improved prognostication based on response ${ }^{13}$; time to plan surgery ${ }^{14}$; and participation in a neoadjuvant or postneoadjuvant clinical trial. ${ }^{15}$ Disadvantages for some patients include fear of disease progression on chemotherapy, ${ }^{16}$ and the psychological impact of leaving a tumor in place rather than its immediate surgical removal and of an adverse prognosis if the tumor does not respond to chemotherapy. Importantly, disease-free and overall survival outcomes are equivalent, whether NAST or surgery is the first treatment modality for operable disease. ${ }^{17}$

Involving patients in the decision about NAST can be challenging due to the complexity and perceived urgency of the decision. This study aimed to develop a DA for women with operable breast cancer who had been offered NAST, and to test the feasibility and acceptability of that DA in clinical practice.

\section{Methods}

\section{Design, Patients, and Setting}

This was a prospective, single-arm, multicenter, longitudinal study. This study received approval from a recognized ethics committee, and all participants provided informed consent prior to any study processes. A detailed description of study methods and DA development has been published previously. ${ }^{18}$ Participants were Australian women aged $\geq 18$ years with a diagnosis of operable invasive breast cancer, and were candidates for NAST of $\geq 3$ months. Exclusion criteria were insufficient English language to complete study questionnaires; inflammatory, metastatic, or inoperable breast cancer; or inability to access the internet and email to complete study requirements.

A DA was developed for this study based on literature review, patient interviews, ${ }^{19}$ a clinician survey, ${ }^{20}$ and the International Patient Decision Aid Standards (IPDAS) Collaboration guidelines (see supplemental eAppendix 1 for the IPDAS checklist, available online with this article at JNCCN.org). ${ }^{4}$ A stakeholder group reviewed the DA, including medical oncologists, surgeons, a psychologist, breast care nurse, consumers, and a breast cancer advocacy group representative. The DA is a 26-page booklet available online or in print. It contains a description of options for adjuvant and neoadjuvant therapy, with advantages and disadvantages, outcome probabilities (pathologic complete response, downstaging, progression on neoadjuvant therapy), graphical and pictorial information, and a values clarification exercise. It is designed for flexibility of use, whether to be read cover-to-cover, or only sections of interest. The Flesch-Kincaid reading level is year 10 , despite consumer input and substantial effort to simplify the language. A full copy of the DA is available at https://www.breastcancertrials.org.au/ file/44/Neoadjuvant-Patient-Decision-Aid. ${ }^{21}$

Participants were recruited by their breast surgeon or medical oncologist, completed the baseline 
questionnaire (assessment 1), and then accessed the DA for on-screen or hard copy review (see supplemental eFigure 1 for study schema). The DA was intended to be given to patients by their surgeon at the time of referral to a medical oncologist, for review prior to that appointment. After the follow-up visit in which a decision was made about undergoing NAST or surgery first, patients received a followup questionnaire (assessment 2). Assessment 3 was between completion of systemic therapy and before surgery (or after surgery and before chemotherapy if surgery was first), and the final questionnaire (assessment 4) was given 12 months after registration. All questionnaires were completed online.

\section{Outcomes}

Co-primary outcomes comprised feasibility of DA use and acceptability to patients and clinicians. Feasibility was defined as the percentage of eligible patients who accessed the DA after being offered participation in the study, which we hypothesized would be $>50 \%$. Patient acceptability was defined as the percentage of participants who would recommend the DA to others in their situation, hypothesized to be $>50 \%$. Investigator acceptability was defined as the percentage of investigators who would use the DA in routine practice, hypothesized to be $>50 \%$. Acceptability and feasibility margins were prospectively defined based on the estimated proportion of patients and investigators required to warrant routine implementation of the DA.

Patient-reported outcome questionnaires were completed by participants at each time point (supplemental eTable 1) and were described in detail previously. ${ }^{18}$ Measures were Decisional Conflict Scale ${ }^{22}$; Information and Involvement Preferences ${ }^{23}$; DecisionMaking Preference Questionnaire ${ }^{24}$; 6-item StateTrait Anxiety Inventory ${ }^{25}$; Distress Thermometer ${ }^{26}$; Fear of Progression Questionnaire-Short Form ${ }^{27}$; Fear of Cancer Recurrence Inventory ${ }^{28}$; custom knowledge questionnaire; Decision Regret Scale ${ }^{29}$; Satisfaction with Decision Scale ${ }^{30}$; and DA feedback. ${ }^{31}$ Investigators were also asked to complete a feedback questionnaire.

\section{Analysis}

Recruitment of 50 participants was planned so that a primary outcome score of $\geq 67 \%$ would provide $80 \%$ power with a one-sided $\alpha$ of $5 \%$ to reject a true rate of $\leq 50 \%$. To provide sufficient numbers for secondary outcome analysis, recruitment was planned to continue until 50 participants had completed assessment 2. We summarized demographic, tumor, and treatment data by mean and standard deviation for continuous data, and by number and frequency for categorical data. Primary outcomes were analysed using tests of proportions and presented with 95\% CIs. Those who did not complete assessment 2 were assumed to have not read the DA. Differences in Decisional Conflict Subscale and Information and Involvement Preferences (detail) were assessed using paired $t$ tests. Change in Information and Involvement Preferences (information type) from baseline was tested using McNemar's test. Linear mixed models were used to examine changes in distress and anxiety, with fixed effects for final treatment choice and time (modeled as a categorical variable) and a subject-level random intercept term. Fear of progression, fear of cancer recurrence, and decisional regret are reported using summary statistics and paired $t$ tests. Agreement in decision control and treatment choice (preferred vs achieved at assessment 2 ) is presented using the $\kappa$ statistic, with scores defined as poor $(<0)$, slight $(0-0.20)$, fair $(0.21-0.40)$, moderate $(0.41-0.60)$, substantial $(0.61-0.80)$, and almost perfect (0.81-0.99). ${ }^{32}$ Change in decisional control is presented using the Bowker test, collapsing categories into shared (collaborative, guided by either patient, doctor, or both equally) versus not shared (only patient or doctor made the final decision). An exploratory analysis of outcomes at assessment 2 was conducted according to whether the participant had read the DA. A $P$ value of $<0.05$ was considered significant for all analyses. Analyses were computed using Stata/IC 13.1 (StataCorp LP).

\section{Results}

Between June 2015 and September 2016, 77 eligible women were offered participation and consented to demographic details being recorded (Figure 1). A total of 59 completed the online consent and the first questionnaire, and accessed the DA (Table 1).

\section{Feasibility and Acceptability of the DA}

The DA was feasible, with 59 of 77 eligible patients accessing it $(76.6 \% ; 95 \% \mathrm{CI}, 67.2-86.1 ; \mathrm{P}<.0001)$. Of those 59 participants, 47 read all or part of the 
Use of a Decision Aid in Breast Cancer

\begin{tabular}{|c|c|c|c|c|c|}
\hline \multicolumn{2}{|c|}{ Prescreened (potentially eligible) } & \multirow[t]{2}{*}{88} & \multirow{2}{*}{\multicolumn{2}{|c|}{ Not offered NAST }} & \\
\hline & & & & & \\
\hline \multicolumn{2}{|l|}{ Offered NAST } & 87 & \multirow{2}{*}{$\begin{array}{l}\text { Not offered DOMINO } \\
\text { Too distressed } \\
\text { Already decided on surgery }\end{array}$} & \multirow[b]{2}{*}{$\begin{array}{l}1 \\
1\end{array}$} & 2 \\
\hline Offered DOMINO & & 85 & & & \\
\hline \multirow[t]{4}{*}{ Entered screening } & & 79 & \multirow{3}{*}{$\begin{array}{l}\text { Declined participation } \\
\text { No internet/computer } \\
\text { Already decided on surgery } \\
\text { Not interested in study/DA }\end{array}$} & \multirow{3}{*}{$\begin{array}{l}2 \\
1 \\
3\end{array}$} & \multirow[t]{2}{*}{6} \\
\hline & & & & & \\
\hline & & & & & \\
\hline & & & \multirow{2}{*}{\begin{tabular}{|l|} 
Ineligible \\
No internet/computer
\end{tabular}} & \multirow[b]{2}{*}{2} & \multirow{2}{*}{2} \\
\hline \multicolumn{2}{|l|}{ Eligible } & 77 & & & \\
\hline & & & \multirow{5}{*}{$\begin{array}{l}\text { Did not enroll } \\
\text { Patient declined participation } \\
\text { Decision made prior to participation } \\
\text { Patient referred to non-study site } \\
\text { Insufficient time to participate } \\
\text { Technical (email not received) }\end{array}$} & \multirow{5}{*}{$\begin{array}{ll} & 1 \\
8 & \\
4 \\
3 \\
2\end{array}$} & 18 \\
\hline \multirow[t]{2}{*}{ Consented online } & & 59 & & & \\
\hline & & & & & \\
\hline \multicolumn{2}{|c|}{ Completed questionnaire set 1} & \multirow[t]{3}{*}{59} & & & \\
\hline & & & & & \\
\hline & & & Nonevaluable & & 8 \\
\hline \multicolumn{2}{|c|}{ Completed questionnaire set 2} & 51 & & & \\
\hline \multicolumn{2}{|c|}{ Completed questionnaire set 3} & 51 & & & \\
\hline \multicolumn{2}{|c|}{ Completed questionnaire set $4^{a}$} & 29 & & & \\
\hline
\end{tabular}

Figure 1. CONSORT diagram.

Abbreviations: DA, decision aid; DOMINO, Decision-Making About Neoadjuvant Therapy for Breast Cancer study; NAST, neoadjuvant systemic therapy.

aAt the data cutoff date, 20 participants had not yet reached their 4 th questionnaire.

DA $(79.7 \% ; 95 \%$ CI, 69.4-89.9; $P<.0001)$. No significant differences were seen in baseline demographics or outcome measure scores between those

\begin{tabular}{|lc|}
\hline Table 1. Participant Characteristics & $\mathbf{n}(\%)$ \\
\hline Variable & 59 \\
\hline Number & $52(8.9)$ \\
\hline $\begin{array}{l}\text { Mean age, y (SD) } \\
\text { Married/De facto }\end{array}$ \\
\hline Single & $48(81.4)$ \\
\hline Highest education level & $11(18.6)$ \\
\hline Postgraduate & $17(28.8)$ \\
\hline Undergraduate & $13(22.0)$ \\
\hline Vocational & $13(22.0)$ \\
\hline High school & $15(25.4)$ \\
\hline Missing & $1(1.7)$ \\
\hline Private health insurance & $51(86.4)$ \\
\hline Yes & $8(13.6)$ \\
\hline No & $10(17.0)$ \\
\hline Health professional & $49(83.0)$ \\
\hline Yes & $36(94.9)$ \\
\hline No & $3(5.1)$ \\
\hline English as first language & \\
\hline Yes & \\
\hline No & \\
\hline
\end{tabular}

who read the DA and those who did not. The DA was acceptable to 41 of 51 participants who completed assessment 2, and they indicated that they would recommend the DA to others in their situation $(82.4 \%$; 95\% CI, 69.5-91.3; $P<.0001) ; 9$ (18\%) were neutral. The DA was acceptable to 16 of 18 site investigators (88.9\%; 95\% CI, 74.4-103.4; $P<.001)$ for routine use.

\section{Patient-Reported Outcomes}

Overall, 51 of 59 participants (86.4\%) chose NAST. Agreement was good between desired (post-DA) and actual treatment ( $\kappa, 0.6 ; 95 \% \mathrm{CI}, 0.290-0.911)$. At baseline, 18 participants were unsure about whether to proceed with NAST or surgery as their first treatment, 6 of whom proceeded with surgery and 12 underwent NAST. Decisional conflict decreased in total and in all subscales (informed, values clarity, support, uncertain, effective) after use of the DA in combination with clinical consultation (all $P$ values $<.002$; Figure 2). The post-DA score was $<25$ in all except the "uncertain" subscale. Patient-reported outcome measure scores are presented in Table 2.

No differences were seen in baseline demographics or assessment 1 outcome scores between those who read the DA and those who did not. At assessment 2, those who read the DA had no difference in decisional conflict, anxiety, distress, or decision control preferences. Those who read the DA had a higher mean knowledge score.

Most patients preferred, and achieved, a shared approach in their decision about NAST (Table 3);

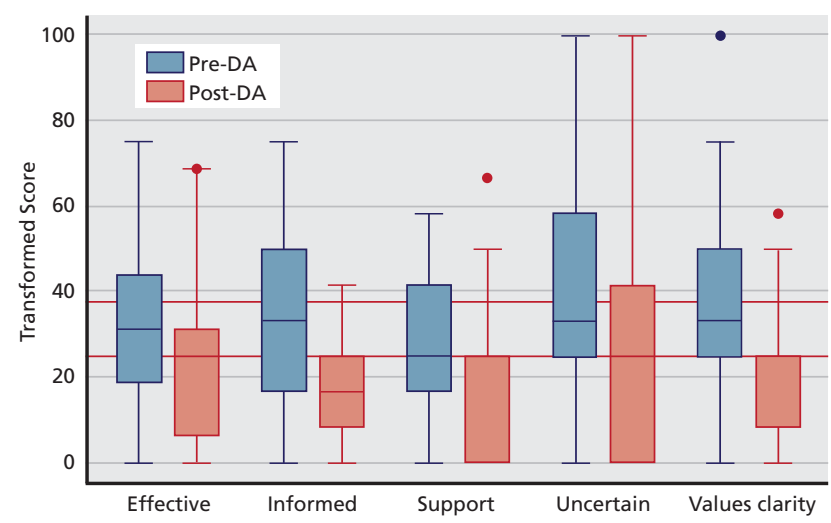

Figure 2. Decisional Conflict Subscales pre- and post-decision aid (DA). Horizontal reference lines at scores of 25 and 37.5. $P<.002$ for all pre/ post-DA comparisons. Boxes represent the interquartile range (25th to 75th); whisker length is 1.5 times the interquartile range above and below the box. Dots represent values outside the whiskers. 
Zdenkowski et al

\begin{tabular}{|c|c|c|c|c|c|}
\hline Measure & $\begin{array}{l}\text { Assessment } 1 \\
(n=59)\end{array}$ & $\begin{array}{c}\text { Assessment } 2 \\
(n=51)\end{array}$ & $\begin{array}{c}\text { Assessment } 3 \\
(n=51)\end{array}$ & $\begin{array}{l}\text { Assessment } 4 \\
(\mathrm{n}=29)^{\mathrm{a}}\end{array}$ & $P$ Value \\
\hline Total decisional conflict, mean (SD) & $33.4 / 100(19.3)$ & $21.0(14.1)$ & - & - & $<.001^{\mathrm{b}}$ \\
\hline Information detail preference, mean (SD) & $8.5 / 10(2.16)$ & $8.6 / 10(2.02)$ & - & - & $.73^{\mathrm{b}}$ \\
\hline Information type & & & & & $.69^{c}$ \\
\hline All information, good and bad & $76.3 \%$ & $72.6 \%$ & - & - & \\
\hline $\begin{array}{l}\text { Additional information only if it is good } \\
\text { news }\end{array}$ & $5.1 \%$ & $3.9 \%$ & - & - & \\
\hline $\begin{array}{l}\text { Only information needed to care for } \\
\text { myself }\end{array}$ & $18.6 \%$ & $23.5 \%$ & - & - & \\
\hline Anxiety, mean (SD) & $55.4 / 100(15.0)$ & $49.4 / 100(14.1)$ & $40.1 / 100(13.0)$ & $36.1 / 100(12.2)$ & $<.001^{\mathrm{d}}$ \\
\hline Distress, mean (SD) & $5.9 / 10(2.9)$ & $5.0 / 10(2.5)$ & $3.2 / 10(2.5)$ & $2.2 / 10(2.5)$ & $<.02^{\mathrm{d}}$ \\
\hline Fear of cancer progression, mean (SD) & - & $33.4 / 60(7.2)$ & $29.9 / 60(7.2)$ & - & $<.001^{\mathrm{b}}$ \\
\hline Fear of cancer recurrence, mean (SD) & - & - & $58.9 / 168(25.0)$ & $57.5 / 168(22.9)$ & $.359^{\mathrm{b}}$ \\
\hline Satisfaction with decision, mean (SD) & - & - & $25.5 / 30(3.6)$ & - & $.005^{\mathrm{e}}$ \\
\hline Decisional regret, mean (SD) & - & - & $16.4 / 100(20.0)$ & $10.0 / 100(15.4)$ & $.0176^{\mathrm{b}}$ \\
\hline Knowledge, mean (SD) & - & $76 \%(12.5)$ & - & - & $<.001^{\mathrm{e}}$ \\
\hline Feedback, mean (SD) & - & $74 \%(8.4)$ & - & - & $<.001^{\mathrm{e}}$ \\
\hline
\end{tabular}

a At the data cutoff, 20 patients had yet to complete their 4th questionnaire.

bPaired $t$ test.

'McNemar's test.

dLinear mixed model.

e $P$ value comparison with prespecified hypothesis.

$86 \%(44 / 51)$ achieved at least as much decisional control as they desired. No significant changes were observed between pre-DA preferred decisional control and actual perceived decisional control $(P=.50)$. Fair agreement was observed between preferred and achieved decisional control at assessment $2(\kappa, 0.32$; 95\% CI, 0.16-0.49). Fear of Cancer Recurrence scores were low in $76.5 \%$ of participants, moderate in $19.6 \%$, and severe in $3.9 \%$ at assessment 2 . At assessment 3 , scores were low in $88.2 \%$ and moderate in the remainder.

\section{Investigator-Reported Outcomes}

Of 24 participating investigators, 18 (75\%; 7/11 medical oncologists and 11/13 surgeons) completed the investigator questionnaire, and 16 (88.9\%) indicated that they would use the DA in routine practice; 16 also indicated that the DA appeared to make the decision easier, or no more difficult, for their patients compared with those who had not used the DA. A total of 14 investigators felt that the DA did not change patients' decisions about NAST, and the remaining 4 felt that the DA increased the likelihood of a patient choosing NAST; 12 noted no change in consultation time, and the remaining 6 noted a mean increase of 7.5 minutes.

\section{Discussion}

This study fills a gap in the breast cancer decision literature by producing a neoadjuvant DA that was not previously available. ${ }^{19}$ Findings show that a DA in this setting was acceptable to most patients and clinicians, and could feasibly be used within routine clinical workflow. Decision-related patient-reported outcome scores improved in the post-DA assessments. These findings are consistent with the literature showing that systematically developed DAs designed for patient use in healthcare decision-making effectively improve decision-related outcomes for a range of general medical treatment decisions ${ }^{6}$ and for breast cancer. ${ }^{\text {? }}$

Previous work has indicated that patients benefit from being at least as involved as they wish to be. ${ }^{9}$ The decision regarding NAST is complex due to the need to consider the implications of a range of individual treatment decisions in the context of distress due to a recent breast cancer diagnosis. Patients in the present study engaged in decision-making despite this inherent complexity. Using the DA did not appear to increase patient anxiety, and, with an additional consultation, appeared to resolve decisional uncertainty. 
Use of a Decision Aid in Breast Cancer

\begin{tabular}{|c|c|c|c|c|c|c|}
\hline Level of Control & $\begin{array}{c}\text { Survey } 1- \\
\text { Preferred } \\
(n=59)\end{array}$ & $\%$ & $\begin{array}{c}\text { Survey } 2- \\
\text { Preferred } \\
(n=51)\end{array}$ & $\%$ & $\begin{array}{c}\text { Survey } 2- \\
\text { Actual } \\
(\mathrm{n}=51)\end{array}$ & $\%$ \\
\hline Doctor makes final decision & 7 & 11.9 & 5 & 9.8 & 6 & 11.8 \\
\hline Doctor makes final decision, seriously considering my opinion & 9 & 15.3 & 11 & 21.6 & 6 & 11.8 \\
\hline Shared & 28 & 47.5 & 26 & 51.0 & 18 & 35.3 \\
\hline I make final decision, seriously considering my doctor's opinion & 15 & 25.4 & 8 & 15.7 & 19 & 37.3 \\
\hline I make final decision & 0 & - & 1 & 2.0 & 2 & 3.9 \\
\hline
\end{tabular}

A DA should be implementable in routine practice to realize the benefits seen in this study. Although the hypothesized threshold values for feasibility and acceptability were based on estimated likelihood of routine uptake, lower (or higher) thresholds might be appropriate depending on individual clinician and patient factors. A minority of patients in this study were neutral regarding whether they would recommend the DA to others in their situation, and only one indicated that she would recommend against it. Most read part or all of the DA once they had accessed it. Reasons for not reading the DA were that they had already made their decision or that the DA was provided too late in their decision-making process. The nonrandomized analysis of outcomes according to whether the participant had read the DA is exploratory and should be interpreted as such.

Study procedures such as the online delivery and pre-DA assessment made access to the DA more difficult than it would be in routine practice, where it would be appropriate to hand the patient a paper copy for immediate review. We suspect that others did not access the DA because it, and the study, were a burden at a time when they were dealing with the impact of their diagnosis and planning for treatment. Therefore, patient uptake in this study may underestimate real-world conditions. Broader reach might be gained if the DA were distributed by breast care nurses as well as clinicians.

At baseline, mean decisional conflict scores were high, consistent with patients delaying or feeling unsure about decision-making. ${ }^{22}$ After use of the DA and a subsequent clinical consultation, decisional conflict scores were consistent with patients implementing their treatment choice. This correlates with a significant decrease in the proportion of participants who reported being unsure about which treatment they preferred. The relative contributions of clinical consultation versus the DA cannot be enumerated with this single-arm study design. Anxiety scores were consistent with participants being in a stressful situation, and did not decrease to population norms until assessment $4 .{ }^{25}$ Lower anxiety in the neoadjuvant group might be explained by patients' expectations of receiving NAST if they were offered that treatment. Alternatively, anxious patients might have elected to undergo surgery first. Lower anxiety might also have been associated with seeing their tumor shrink and with getting a feared treatment "over and done with." A decision to have surgery upfront might also have been seen as contradicting the perceived medical recommendation in patients who had been referred for NAST.

Scores for fear of cancer progression were similar to the mean score of $60.6(\mathrm{SD}, 24.6)$ reported by historical breast cancer controls. ${ }^{28}$ This suggests that these women were no more worried than the average patient with breast cancer about the impact of the tumor becoming larger or developing distant metastases while on treatment. Mean decisional regret at assessment 3 was comparable with historical patient data indicating low levels of regret related to the decision about NAST. ${ }^{29}$ The strength of clinical recommendation for NAST will depend on the individual patient and tumor characteristics. Clinical equipoise was not expected regarding whether NAST or surgery was the best initial approach for individual patients, nor was the DA expected to change patients' minds. Results support this, with no significant changes in preferred treatment following use of the DA. Only 18 patients reported being unsure about their preferred treatment schedule pre-DA, which may represent clinical influence toward treatment decisions. Of those $18 \mathrm{pa}-$ tients, 6 chose surgery, whereas 2 of the 41 who were sure about their decision at baseline chose surgery. Unsure patients may choose the normative behavior 
Zdenkowski et al

of surgery first, or the DA may influence unsure patients toward surgery. It is possible that the DA was not balanced, although most patients and clinicians perceived it to be.

This study is limited by its relatively small sample size and single-arm design, although a single-arm design was felt to provide adequate confidence in the DA's safety and acceptability. Positive end points allow broad implementation of this DA, given the large amount of existing literature on the efficacy of these tools. We cannot derive the relative contribution of the DA versus clinical consultations, independent pursuit of information, discussion with family and friends, or natural history. The study population was well educated, which may reduce the generalizability to a less educated group. The DA was written at year 10 reading level-higher than the recommended year 8 level, which is a common issue in patient information material. ${ }^{33}$ Strengths include the evidence-based, systematic development process and the use of validated patient-reported outcome measures. The DA was used successfully in a variety of settings as part of this study, supporting its ability to be implemented in routine clinical care.

Because DA uptake is typically low, ${ }^{34}$ implementation implications must be considered. A minority of investigators reported a small increase in consultation time when they used the DA, with no impact on their intention to use the DA, suggesting that they considered the benefits of the DA worth the additional consultation time. The document was intended to be reviewed in detail outside of the con- sultation to reduce impact on consultation time. The DA is scalable through electronic distribution. The intervention will be made freely available to encourage sustained uptake.

\section{Conclusions}

A DA for women with operable breast cancer who were offered NAST was acceptable to clinicians and patients. It was feasible to integrate into patient care pathways, ideally after an appointment with a surgeon and before consultation with a medical oncologist for a final treatment decision. Improvements in decision-related outcomes were seen, without evidence of adverse effects. Implementation strategies are warranted to ensure optimal use in routine patient care.

\section{Acknowledgments}

This study was a collaboration between the Australia and New Zealand Breast Cancer Trials Group (ANZBCTG) and the Psycho-oncology Co-operative Research Group. The ANZBCTG Consumer Advisory Panel reviewed the content of the DA and the study. It was centrally coordinated by the ANZBCTG. The authors wish to thank the participants, investigators, and study coordinators for their contributions. The DA was developed with the assistance of a multidisciplinary team, including consumers, clinicians, researchers, and the Breast Cancer Network Australia (BCNA).

\section{References}

1. Barry MJ, Edgman-Levitan S. Shared decision making-pinnacle of patient-centered care. N Engl J Med 2012;366:780-781.

2. Institute of Medicine. Crossing the Quality Chasm: A New Health System for the 21st Century. Washington, DC: National Academies Press; 2001.

3. Charles C, Gafni A, Whelan T. Shared decision-making in the medical encounter: what does it mean? (or it takes at least two to tango). Soc Sci Med 1997;44:681-692.

4. Volk R, Llewellyn-Thomas $H$, Stacey D, et al. Ten years of the International Patient Decision Aid Standards Collaboration: evolution of the core dimensions for assessing the quality of patient decision aids. BMC Med Inform Decis Mak 2013;13:S1.

5. McCaffery KJ, Smith S, Shepherd HL, et al. Shared decision making in Australia in 2011. Z Evid Fortbild Qual Gesundhwes 2011;105:234-239.

6. Stacey D, Legare F, Lewis $K$, et al. Decision aids for people facing health treatment or screening decisions. Cochrane Database Syst Rev 2017;4:Cd001431.

7. Zdenkowski N, Butow P, Tesson S, Boyle F. A systematic review of decision aids for patients making a decision about treatment for early breast cancer. Breast 2016;26:31-45.

8. Kaufmann M, von Minckwitz G, Mamounas EP, et al. Recommendations from an international consensus conference on the current status and

future of neoadjuvant systemic therapy in primary breast cancer. Ann Surg Oncol 2012;19:1508-1516.

9. Brown R, Butow P, Wilson-Genderson M, et al. Meeting the decisionmaking preferences of patients with breast cancer in oncology consultations: impact on decision-related outcomes. J Clin Oncol 2012;30:857-862.

10. Mougalian SS, Soulos $P R$, Killelea BK, et al. Use of neoadjuvant chemotherapy for patients with stage I to III breast cancer in the United States. Cancer 2015;121:2544-2552.

11. Shin $\mathrm{HC}$, Han W, Moon HG, et al. Breast-conserving surgery after tumor downstaging by neoadjuvant chemotherapy is oncologically safe for stage III breast cancer patients. Ann Surg Oncol 2013;20:2582-2589.

12. Read RL, Flitcroft $K$, Snook KL, et al. Utility of neoadjuvant chemotherapy in the treatment of operable breast cancer. ANZ J Surg 2015;85:315-320.

13. Cortazar P, Zhang L, Untch M, et al. Pathological complete response and long-term clinical benefit in breast cancer: the CTNeoBC pooled analysis. Lancet 2014;384:164-172.

14. Monrigal E, Dauplat J, Gimbergues $P$, et al. Mastectomy with immediate breast reconstruction after neoadjuvant chemotherapy and radiation therapy. A new option for patients with operable invasive breast cancer. Results of a 20 years single institution study. Eur J Surg Oncol 2011;37:864870. 
15. DeMichele A, Yee D, Berry DA, et al. The neoadjuvant model is still the future for drug development in breast cancer. Clin Cancer Res 2015;21:2911-2915.

16. Caudle AS, Gonzalez-Angulo AM, Hunt KK, et al. Impact of progression during neoadjuvant chemotherapy on surgical management of breast cancer. Ann Surg Oncol 2011;18:932-938.

17. Mauri D, Pavlidis N, Ioannidis JP. Neoadjuvant versus adjuvant systemic treatment in breast cancer: a meta-analysis. J Natl Cancer Ins 2005;97:188-194.

18. Zdenkowski N, Butow P, Hutchings E, et al. A decision aid for women considering neoadjuvant systemic therapy for operable invasive breast cancer: development and protocol of a phase II evaluation study (ANZ1301 DOMINO). JMIR Res Protoc 2016;5:e88.

19. Zdenkowski N, Butow $P$, Fewster $S$, et al. Exploring decision making about neoadjuvant chemotherapy for breast cancer. Breast J 2016;22:133-134.

20. Zdenkowski N, Butow P, Mann GB, et al. A survey of Australian and New Zealand clinical practice with neoadjuvant systemic therapy for breast cancer. Intern Med J 2016;46:677-683.

21. Joseph-Williams N, Newcombe R, Politi M, et al. Toward minimum standards for certifying patient decision aids: a modified delphi consensus process. Med Decis Making 2014;34:699-710.

22. O'Connor AM. Validation of a decisional conflict scale. Med Decis Making 1995;15:25-30.

23. Cassileth BR, Zupkis RV, Sutton-Smith K, March V. Information and participation preferences among cancer patients. Ann Intern Med 1980;92:832-836

24. Degner LF, Sloan JA, Venkatesh P. The Control Preferences Scale. Can J Nurs Res 1997;29:21-43.
25. Marteau TM, Bekker H. The development of a six-item short-form of the state scale of the Spielberger State-Trait Anxiety Inventory (STAI). Br J Clin Psychol 1992;31:301-306.

26. Hegel MT, Collins ED, Kearing S, et al. Sensitivity and specificity of the Distress Thermometer for depression in newly diagnosed breast cancer patients. Psychooncology 2008; 17:556-560.

27. Mehnert A, Berg P, Henrich G, Herschbach P. Fear of cancer progression and cancer-related intrusive cognitions in breast cancer survivors. Psychooncology 2009;18:1273-1280.

28. Simard S, Savard J. Fear of Cancer Recurrence Inventory: development and initial validation of a multidimensional measure of fear of cancer recurrence. Support Care Cancer 2009;17:241-251

29. Brehaut JC, O'Connor AM, Wood TJ, et al. Validation of a decision regret scale. Med Decis Making 2003;23:281-292.

30. Holmes-Rovner M, Kroll J, Schmitt N, et al. Patient satisfaction with health care decisions: the satisfaction with decision scale. Med Decis Making 1996;16:58-64.

31. Juraskova I, Butow P, Lopez A, et al. Improving informed consent: pilot of a decision aid for women invited to participate in a breast cancer prevention trial (IBIS-II DCIS). Health Expect 2008;11:252-262.

32. Viera AJ, Garrett JM. Understanding interobserver agreement: the kappa statistic. Fam Med 2005;37:360-363.

33. Stossel LM, Segar N, Gliatto P, et al. Readability of patient education materials available at the point of care. J Gen Intern Med 2012;27:11651170.

34. Elwyn G, Scholl I, Tietbohl C, et al. "Many miles to go ...”: a systematic review of the implementation of patient decision support interventions into routine clinical practice. BMC Med Inform Decis Mak 2013;13:S14.

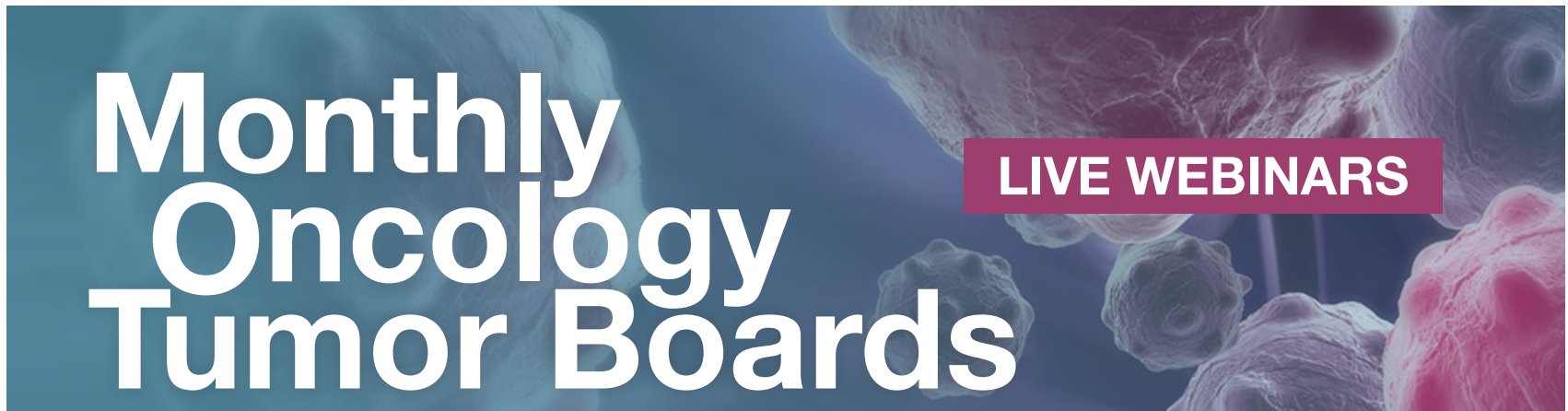

A Multidisciplinary Approach to Individualized Patient Care

\section{Prostate Cancer}

Thursday, April 26, 2018 • 11:00 AM - 12:00 PM EST

Richard J. Lee, MD, PhD, and Adam Feldman, MD, MPH Massachusetts General Hospital Cancer Center

\section{Chronic Lymphocytic Leukemia (CLL)}

Monday, May 14, 2018 • 11:30 AM - 12:30 PM EDT

Jennifer Brown, MD, PhD, and Matthew Davids, MD, MMSc

Dana-Farber Cancer Institute

\section{See JNCCN.org for supplemental online content.}

This program has been approved for AMA PRA Category 1 Credit" for physicians and will award contact hours for nurses, pharmacists, and other health care

professionals. NCCN will also seek approval for case manager clock hours.

This activity is supported by educational grants from Astellas and Medivation, Inc., a Pfizer Company; AstraZeneca; Celgene Corporation; Gilead Sciences, Medical Affairs;

Lilly; Novartis; Pharmacyclics LLC, an AbbVie Company and Janssen Biotech, Inc.; Taiho Oncology; and Takeda Oncology. This activity is supported by independent

educational grants from AbbVie and Boehringer Ingelheim Pharmaceuticals, Inc. This educational activity is supported by a medical education grant from Exelixis, Inc. 